\title{
Sélection de deux caractères chez une espèce de Trichogrammes, efficacité parasitaire des souches obtenues (Hym Trichogrammatidae)
}

\author{
B Pintureau \\ INSA-INRA, Laboratoire associé Biologie 406, 20 avenue A Einstein, 69621 Villeurbanne cedex, France
}

(Reçu le 17 mai 1990; accépté le 8 avril 1991)

\begin{abstract}
Résumé - Deux caractères ont été séparément ou simultanément sélectionnés chez $T$ brassicae Bezdenko, la fécondité et la "capacité de recherche de l'hôte». Le 1 er a connu une amélioration allant jusqu'à $71 \%$ contrairement au $2^{\theta}$. Cet échec peut s'expliquer par une surestimation de l'héritabilité ou par un manque de standardisation de la mesure du caractère. L'augmentation de la fécondité des souches n'a pas eu d'influence nette sur l'efficacité parasitaire appréciée dans des serres.

Nous discutons les avantages et inconvénients de diverses stratégies envisageables pour la lutte biologique : maintien, comme actuellement, de la plus grande variabilité génétique possible; sélection de certains caractères au laboratoire; sélection de l'efficacité parasitaire dans des conditions naturelles ou semi-naturelles.
\end{abstract}

génétique / lutte biologique / sélection / Hyménoptère / Trichogramme / fécondité / capacité de recherche de l'hôte

Summary - Selection of two characteristics in a Trichogramma species: parasitic efficacy of the obtained strains (Hym: Trichogrammatidae). Two characteristics, fecundity and "host-finding capacity", were selected in T brassicae Bezdenko over 5 generations. Three experiments were carried out. The first experiment concerned only fecundity, selected according to 2 methods (mass selection and "by strain" selection) with a pressure of $50 \%$. It allowed fecundity to be improved by up to 52 or $71 \%$. The second experiment concerned only the "host-finding capacity", selected under the same conditions. It did not allow improvement of character. This failure can be explained by an overestimation of the heritability or by a lack of standardization of the measurements. The third experiment concerned the 2 characteristics, separately or simultaneously (by means of an index) selected according to the "by strains" method with a pressure of $66 \%$. The characters were not improved and this failure can be explained by a loss of fecundity variability during the rearing period between the 1st and 3rd experiment. The parasitic efficacy of the strains both selected or not was tested in greenhouses planted with corn where corn borers were released. It was concluded that the increase in fecundity had no clear influence on the biological control. The advantages and disadvantages of different biological control strategies have been discussed: maintaining, as is the current practice, the greatest possible genetic variability; selection of some characteristics in the laboratory; selection of the parasitic efficacy under natural or seminatural conditions. Methodologies have been suggested and compared.

genetics / biological control / selection / Hymenoptera / Trichogramma / fecundity / host-finding capacity 


\section{INTRODUCTION}

Les premiers projets de sélection chez les Trichogrammes (parasites oophages utilisés en lutte biologique) sont dus à Urquijo et datent de 1944. Cet auteur souhaitait alors améliorer la fécondité, l'«instinct au parasitisme», la viabilité et le taux sexuel. II n'a en fait effectué que des tris de lignées fondées par un couple, ceci au cours de plusieurs générations, selon le critère "taux de parasitisme». En 1946 et 1950, il a mené les mêmes opérations sur la fécondité et l'ovotropisme (ce qu'il appelait auparavant «instinct au parasitisme" et que nous appelons plutôt maintenant «capacité de recherche de l'hôte»). D'après lui, la sélection aurait été efficace, bien que cela reste à démontrer. Aucune comparaison sérieuse entre populations n'a cependant été effectuée hors du laboratoire.

Plus tard, en 1974, Ashley et al ont utilisé des méthodes plus rigoureuses pour sélectionner la tolérance à la température de $43^{\circ} \mathrm{C}$, la capacité de locomotion et ces 2 caractères à la fois. Dans des cages placées sur le terrain, les populations modifiées ne se sont pourtant pas montrées plus efficaces que les témoins, voire partois moins efficaces.

Ensuite, Ram et Sharma (1977) ont tenté de sélectionner la fécondité et le taux sexuel durant 16 générations. Le premier caractère a ainsi pu être amélioré mais pas le second.

Bigler et al ont enfin mené, en 1982, une sélection dans une serre pour augmenter la "rapidité à trouver des hôtes". II s'agit alors d'une tentative d'amélioration en milieu seminaturel, portant sur un caractère complexe qui en intègre plusieurs autres. Elle s'est semble-t-il conclue par un succès.

Chez d'autres Hyménoptères parasites, des sélections ont porté sur la tolérance à un climat donné, le taux sexuel, la fécondité, le pourcentage de mâles stériles, la résistance aux insecticides (Wilkes, 1942, 1947; Simmonds, 1947; Pielou et Glasser, 1951; Robertson, 1957; White et al, 1970; Szmidt, 1972). Aucun essai d'efficacité (mis à part celui peu concluant de Szmidt, 1972) n'est cependant connu à la suite de ces tentatives d'amélioration.

Nous présentons ici les résultats de quelques sélections menées au laboratoire sur 2 caractères qui semblent impliqués dans la réussite de la lutte biologique. Plusieurs méthodes de sélection ont été testées. L'efficacité parasitaire des souches ainsi modifiées a ensuite été appréciée dans des serres plantées de maïs infesté de Pyrales (hôtes des Trichogrammes).

\section{MATÉRIEL ET MÉTHODE}

L'étude a été effectuée chez Trichogramma brassicae Bezdenko, dont $T$ maidis Pintureau et Voegelé est un synonyme (Pintureau, 1987). Le matériel est constitué de souches fondées chacune à partir de quelques individus provenant de Moldavie soviétique, de Hongrie, de Bulgarie et, après recapture dans des zones de lâcher, de République fédérale allemande, Suisse et France. Celles-ci ont été élevées sur Ephestia kuehniella Zeller (Lép Pyralididae).

Des héritabilités significatives ont été calculées, par la méthode de la régression parents-enfants, pour la fécondité à $25^{\circ} \mathrm{C}\left(\mathrm{h}^{2}=0,45 \pm 0,15\right)$, pour la "capacité de recherche de l'hôte" $\left(h^{2}=0,46 \pm 0,16\right)$ et pour le nombre d'arrêts au cours d'une marche de $10 \mathrm{~min}$. Les autres caractères étudiés ont donné des valeurs non significatives (fécondité à $17^{\circ} \mathrm{C}$ et à $30^{\circ} \mathrm{C}$, taux sexuel, longévité, distance parcourue à la marche en $10 \mathrm{~min}$ ) (Babi, 1985; Pintureau, 1988).

Nous avons choisi de sélectionner la fécondité à $25^{\circ} \mathrm{C}$ (il s'agit en fait du nombre d'œufs d'E kuehniella parasités au cours des 7 premiers jours de ponte : voir Pintureau et al, 1981) et la "capacité de recherche de l'hôte" (Babi et Pintureau, 1984; Babi, 1985).

$\mathrm{Ce}$ dernier caractère, dont nous ne connaissons par la valeur dans la nature, a été mesuré de la manière suivante : nombre d'œufs d'E kuehniella touchés en $10 \mathrm{~min}$ par une femelle introduite dans une cage en plastique transparent de $30 \times 20 \times 2 \mathrm{~cm}$; les hôtes sont collés sur une feuille de papier blanc placée sur le plancher de la cage, ils sont au nombre de 81, séparés les uns des autres par $2 \mathrm{~cm}$; la femelle fécondée, nourrie, n'ayant jamais pondu, est introduite dans la cage à une distance de $12 \mathrm{~cm}$ du premier hôte; après chaque mesure concernant une femelle, la cage est lavée à l'alcool et les hôtes changés. L'observation constante nécessaire à la mesure du caractère ne nous a pas permis d'utiliser une enceinte climatisée, aussi la température $\left(22\right.$ à $24^{\circ} \mathrm{C}$ ) et l'éclairage (stores baissés, un tube néon allumé) sont-ils ceux du laboratoire au mois de mai à Antibes (France).

Nous avons aussi sélectionné l'ensemble “fécondité - capacité de recherche de l'hôte" à l'aide d'un index (l) qui tient compte de la valeur et de la variance des 2 caractères, mais également de leur héritabilité, de leur corrélation et de leur importance économique (d'après Falconer, 1974). Les coefficients d'importance économique sont calculés ici d'après les pourcentages de parasitisme obtenus en serre avec des populations sélectionnées pour l'un ou l'autre caractère.

$$
I=P_{F}+W \cdot P_{C R}
$$




$$
P_{F}=\frac{F-F_{F O}}{\sigma_{F}}
$$

$\left(P_{C R}\right.$ est calculé de la même manière; $F$ et $C R$ : valeurs phénotypiques de la fécondité et de la capacité de recherche de ('hôte; $\sigma$ : écart type à la Fo)

$$
W=\frac{\sigma^{2} \cdot \operatorname{Cov}{ }_{H . C R}-\operatorname{Cov}_{H . F} \cdot \operatorname{Cov}_{F . C R}}{\sigma_{C R}^{2} \cdot \operatorname{Cov}_{H . F}-\operatorname{Cov}_{H . C H} \cdot \operatorname{Cov}_{F . C R}}
$$

( $H$ : valeurs additives)

$\operatorname{Cov}_{H . F}=\omega_{F} \cdot h^{2} F \cdot \sigma^{2}{ }_{F}+\omega_{C R} \cdot r_{A} \cdot h_{F} \cdot h_{C R} \cdot \sigma_{F} \cdot \sigma_{C R}$

$\operatorname{Cov}_{H . C A}=\omega_{C R} \cdot h^{2} C R^{\cdot} \sigma^{2} C R+\omega_{F} \cdot r_{A} \cdot h_{F} \cdot h_{C R} \cdot \sigma_{F} \cdot \sigma_{C R}$

$\operatorname{Cov}_{F . C R}=r_{P} \cdot \sigma_{F} \cdot \sigma_{C R}$

$$
r_{A}=\frac{\operatorname{Cov}_{F \cdot C R}}{\sqrt{\sigma^{2} F \cdot \sigma^{2} C R}}
$$

$\omega=$ importance économique du caractère; $h^{2}=$ héritabilité; $r_{P}=$ corrélation phénotypique entre les caractères.

Les sélections ont été effectuées de 2 manières différentes : sélections massales selon la formule la plus banale (tableau I), sélections bien moins classiques que nous avons appelées "par lignées" (Babi et Pintureau, 1984; Babi, 1985) (tableau II). Ces dernières

\begin{tabular}{|c|c|c|}
\hline $\begin{array}{l}F-3 \\
F-2 \\
F-1\end{array}$ & $\begin{array}{l}20 \text { souches } \\
\text { mélange } \\
\text { des souches }\end{array}$ & \\
\hline $\begin{array}{l}F_{0} \\
F_{1} \\
F_{2} \\
F_{3} \\
F_{4} \\
F_{5}\end{array}$ & $\begin{array}{l}100 \mathrm{~lm} \\
100 \mathrm{~lm} \\
100 \mathrm{~lm} \\
100 \mathrm{~lm} \\
100 \mathrm{~lm} \\
100 \mathrm{~lm}\end{array}$ & $\begin{array}{l}50 \text { ls } \\
50 \text { is } \\
50 \text { is } \\
50 \text { is } \\
50 \text { is } \\
\text { fondation de la } \\
\text { "population sélectionnée» }\end{array}$ \\
\hline
\end{tabular}

Tableau I. Schéma de sélection massale utilisé chez $T$ brassicae. La pression est de $50 \%$.

Im : individus mesurés; Is : individus sélectionnés.

constituent un compromis entre la sélection intrafamiliale et la sélection massale qui doit théoriquement s'opposer à la diminution de la variabilité des caractères non sélectionnés. Il offre par contre un inconvénient en créant d'éventuels effets d'hétérosis à chaque génération qui peuvent fausser l'interprétation des résultats (l'amélioration du caractère est, dans ce cas, due à la fois à la sélection et à l'hétérosis). La pression a été appliquée durant 5 générations successives dans le sens d'une augmentation de la valeur des caractères.

Tableau II. Schéma de sélection «par lignées" utilisé chez T brassicae. La pression est de 50\% (chiffres hors parenthèses) ou de $66 \%$ (chiffres entre parenthèses).

\section{$\begin{array}{llllllllllllllllllllll}F-1 & 20 & 1 & 2 & 3 & 4 & 5 & 6 & 7 & 8 & 9 & 10 & 11 & 12 & 13 & 14 & 15 & 16 & 17 & 18 & 19 & 20\end{array}$ souches}

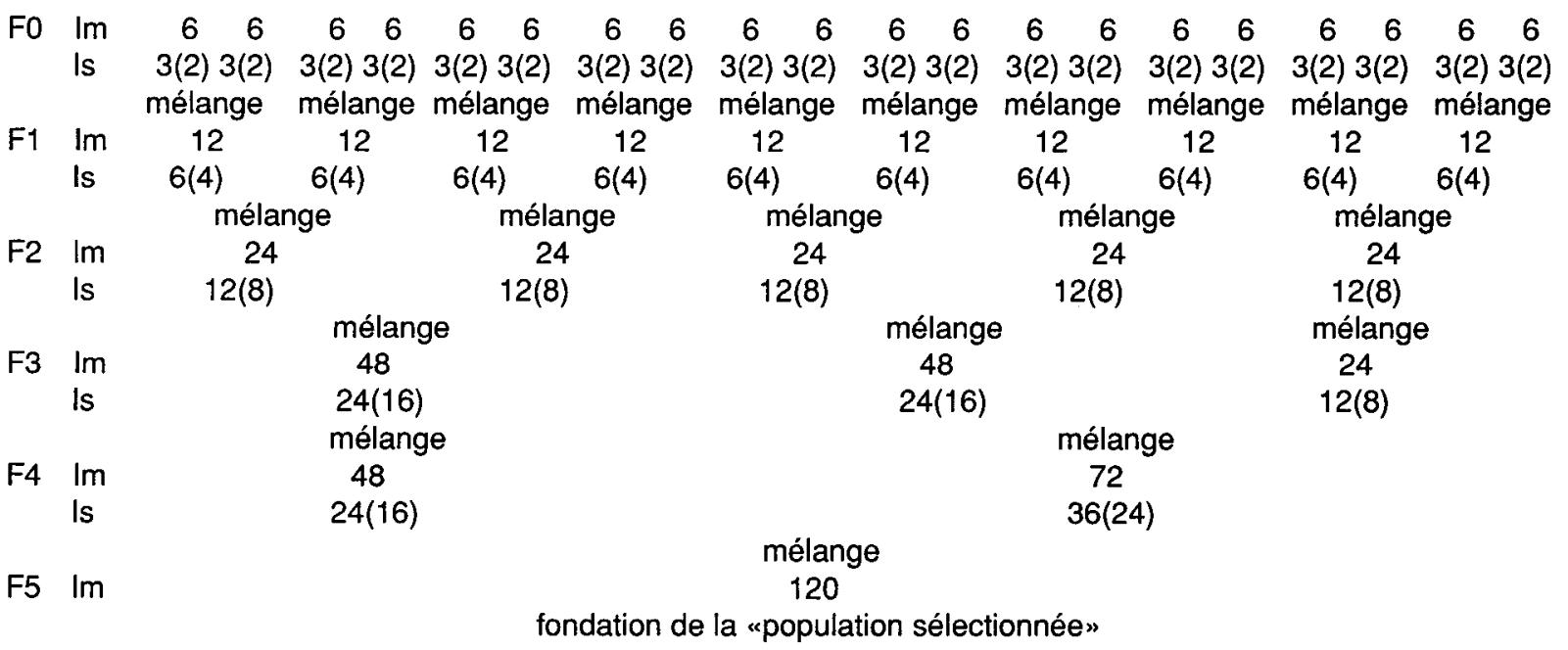


Les caractères sont mesurés sur des femelles fécondées isolées. Même lorsque la fécondité n'est pas prise en compte, la ponte a lieu de façon isolée et ce n'est que quelques jours avant l'émergence que la progéniture des femelles retenues lors de la sélection est regroupée (plus ou moins partiellement pour la sélection "par lignées"). Les parasitoïdes se croisent ainsi librement (nous ne choisissons donc pas les partenaires). Les nouveaux isolements ont lieu un jour après l'émergence (Babi, 1985).

Nous avons d'abord sélectionné la fécondité avec une pression de $50 \%$ (c'est-à-dire que la moitié des individus est rejetée), de 2 manières différentes (en masse et "par lignées"). Nous avons ensuite sélectionné la "capacité de recherche de l'hôte" avec la même pression, toujours de 2 manières différentes. Nous avons enfin sélectionné, uniquement «par lignées" et avec une pression de $66 \%$ (c'est-à-dire que les $2 / 3$ des individus sont rejetés), la fécondité seule, la "capacité de recherche de l'hôte" seule et les 2 caractères simultanément (index).

La comparaison de l'efficacité des populations sélectionnées, entre elles et à celle d'un témoin, a été effectuée dans 3 serres contiguës ( 3 serres de $24 \mathrm{~m}^{2}$ pour les essais "sélections avec une pression de $50 \%$ ", 3 serres de $10,5 \mathrm{~m}^{2}$ pour l'essai "sélections avec une pression de $66 \%$ ) plantées de maiis infesté par Ostrinia nubilalis (Hübner) (Lép Pyralididae). Un nombre égal de femelles et de mâles d'O nubilalis a été lâché dans chaque serre ( 21 couples pour l'essai «sélections de la fécondité avec une pression de $50 \%$, 29 pour l'essai "sélections de la capacité de recherche de l'hôte avec une pression de 50\%", 92 pour l'essai "sélections avec une pression de 66\%"), de façon échelonnée sur 12-20 j. Trois lâchers de Trichogrammes ont été réalisés, espacés de $5 \mathrm{j}$ (essais

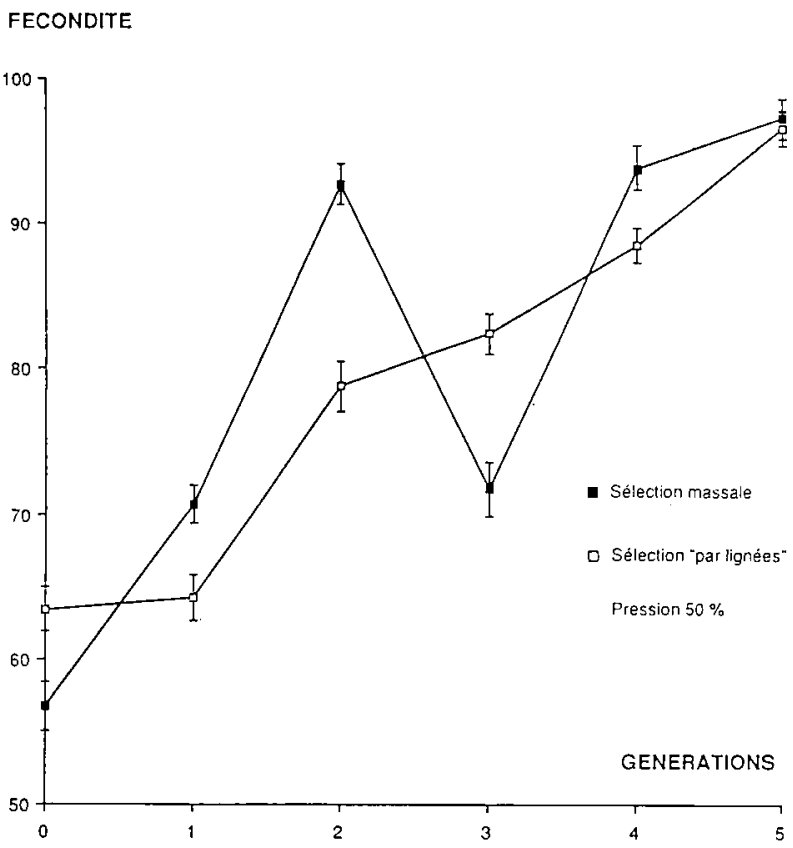

Fig 1. Sélection de la fécondité chez $T$ brassicae en 5 générations. "sélections avec une pression de $50 \%$ ") ou de 4 puis $7 \mathrm{j}$ (essai "sélections avec une pression de 66\%"). La dose de Trichogrammes correspond à 140 000/ha (essai «sélections de la fécondité avec une pression de $50 \%$ ") ou à 200 000/ha (autres essais).

Chaque serre a été divisée en 6 (essai «sélections de la fécondité avec une pression de $50 \%$ ") ou 8 parcelles (autres essais) permettant de tester la signification des différences enregistrées entre les taux de parasitisme (test "t" et test " $U$ " non paramétrique). Ces comparaisons concernent d'abord les sélections "fécondité" (pression de 50\%) et un témoin non sélectionné (Babi, 1985), ensuite les sélections «capacité de recherche de l'hôte" (pression $50 \%$ ) et un témoin non sélectionné (Babi, 1985), enfin les sélections "fécondité", «capacité de recherche de l'hôte» et «index» (pression 66\%).

\section{RÉSULTATS}

\section{Sélections}

\section{Fécondité (pression de 50\%)}

La sélection a été apparemment efficace (fig 1 et tableau III) puisque les réponses obtenues (pente des droites de régression) sont égales aux réponses attendues (iøh $h^{2}$ où $i$ est l'intensité de la sélection) en tenant compte des erreurs standards. La corrélation fécondité-génération est significative au seuil de $5 \%$ pour la sélection massale et au seuil de $1 \%$ pour la sélection "par lignées".

L'amélioration est de $71 \%$ dans un cas (sélection massale) et de $52 \%$ dans l'autre (sélection "par lignées») (Babi et Pintureau, 1984; Babi, 1985). Nous aboutissons à des valeurs comparables avec les 2 types de sélections et cet écart n'est dû qu'à des points de départ différents. Par ailleurs, ces résultats ne permettent de déceler aucun effet d'hétérosis attribuable à la sélection "par lignées".

Nous remarquons que l'amélioration du caractère est très rapide, et peut-être trop si l'on en juge d'après les résultats obtenus chez d'autres espèces. Notre interprétation des observations demanderait donc à être confirmée, bien que l'évolution de la fécondité après le relâchement de la sélection tende à la valider (fig 2) : la valeur du caractère diminue lentement et régulièrement jusqu'à la $35^{\mathrm{e}}$ génération, et certainement audelà, mais les mesures n'ont pas été poursuivies (Babi, 1985). 
Tableau III. Valeur des caractères sélectionnés durant 5 générations chez $T$ brassicae. L'effectif figure entre parenthèses.

\section{Générations}

\begin{tabular}{|c|c|c|c|c|c|c|c|}
\hline Caractère & Pression & $\begin{array}{c}\text { Mode } \\
\text { de sélection }\end{array}$ & 0 & 1 & 2 & 3 & 4 \\
\hline
\end{tabular}

\begin{tabular}{|c|c|c|c|c|c|c|c|c|}
\hline & & Massal & $\begin{array}{c}56,7 \pm 1,7 \\
(99)\end{array}$ & $\begin{array}{c}70,7 \pm 1,3 \\
(96)\end{array}$ & $\begin{array}{c}92,7 \pm 1,4 \\
(98)\end{array}$ & $\begin{array}{c}71,7 \pm 1,9 \\
(84)\end{array}$ & $\begin{array}{c}93,8 \pm 1,5 \\
(99)\end{array}$ & $\begin{array}{c}97,2 \pm 1,4 \\
(100)\end{array}$ \\
\hline & & «Par lignées» & $\begin{array}{c}63,4 \pm 1,5 \\
(109)\end{array}$ & $\begin{array}{c}64,2 \pm 1,6 \\
(117)\end{array}$ & $\begin{array}{c}78,8 \pm 1,7 \\
(117)\end{array}$ & $\begin{array}{c}82,4 \pm 1,4 \\
(115)\end{array}$ & $\begin{array}{c}88,5 \pm 1,2 \\
(118)\end{array}$ & $\begin{array}{c}96,5 \pm 1,2 \\
(120)\end{array}$ \\
\hline $\begin{array}{l}\text { «Capacité } \\
\text { de } \\
\text { recherche }\end{array}$ & $50 \%$ & Massal & $\begin{array}{c}1,08 \pm 0,19 \\
(106)\end{array}$ & $\begin{array}{c}1,84 \pm 0,28 \\
(106)\end{array}$ & $\begin{array}{c}0,82 \pm 0,13 \\
(106)\end{array}$ & $\begin{array}{c}1,19 \pm 0,18 \\
(100)\end{array}$ & $\begin{array}{c}0,72 \pm 0,13 \\
(132)\end{array}$ & $\begin{array}{c}1,47 \pm 0,23 \\
(106)\end{array}$ \\
\hline $\begin{array}{l}\text { de } \\
\text { l'hôte" }\end{array}$ & & "Par lignées" & $\begin{array}{c}1,42 \pm 0,23 \\
(120)\end{array}$ & $\begin{array}{c}1,10 \pm 0,19 \\
(120)\end{array}$ & $\begin{array}{c}0,92 \pm 0,17 \\
(120)\end{array}$ & $\begin{array}{c}2,53 \pm 0,29 \\
(120)\end{array}$ & $\begin{array}{c}1,98 \pm 0,23 \\
(120)\end{array}$ & $\begin{array}{c}1,83 \pm 0,25 \\
(122)\end{array}$ \\
\hline Fécondité & $66 \%$ & «Par lignées» & $\begin{array}{c}45,2 \pm 1,3 \\
\quad(120)\end{array}$ & $\begin{array}{c}71,5 \pm 1,5 \\
(120)\end{array}$ & $\begin{array}{l}45,4 \pm 1,0 \\
(120)\end{array}$ & $\begin{array}{c}64,9 \pm 1,3 \\
(120)\end{array}$ & $\begin{array}{c}63,7 \pm 1,1 \\
(120)\end{array}$ & $\begin{array}{c}59,8 \pm 1,3 \\
(119)\end{array}$ \\
\hline $\begin{array}{l}\text { «Capacité de } \\
\text { recherche } \\
\text { de l'hôte» }\end{array}$ & $66 \%$ & «Par lignées» & $\begin{array}{c}2,42 \pm 0,27 \\
(120)\end{array}$ & $\begin{array}{c}1,19 \pm 0,19 \\
(120)\end{array}$ & $\begin{array}{c}2,08 \pm 0,27 \\
(120)\end{array}$ & $\begin{array}{c}0,71 \pm 0,15 \\
(120)\end{array}$ & $\begin{array}{c}2,24 \pm 0,23 \\
(120)\end{array}$ & $\begin{array}{c}2,36 \pm 0,28 \\
(114)\end{array}$ \\
\hline Index & $66 \%$ & “Par lignées» & $\begin{array}{l}0 \pm 0,10 \\
(120)\end{array}$ & $\begin{array}{c}0,14 \pm 0,12 \\
(120)\end{array}$ & $\begin{array}{c}-0,35 \pm 0,10 \\
(120)\end{array}$ & $\begin{array}{l}0,72 \pm 0,12 \\
(120)\end{array}$ & $\begin{array}{c}1,03 \pm 0,11 \\
(120)\end{array}$ & $\begin{array}{c}0,62 \pm 0,12 \\
(120)\end{array}$ \\
\hline
\end{tabular}

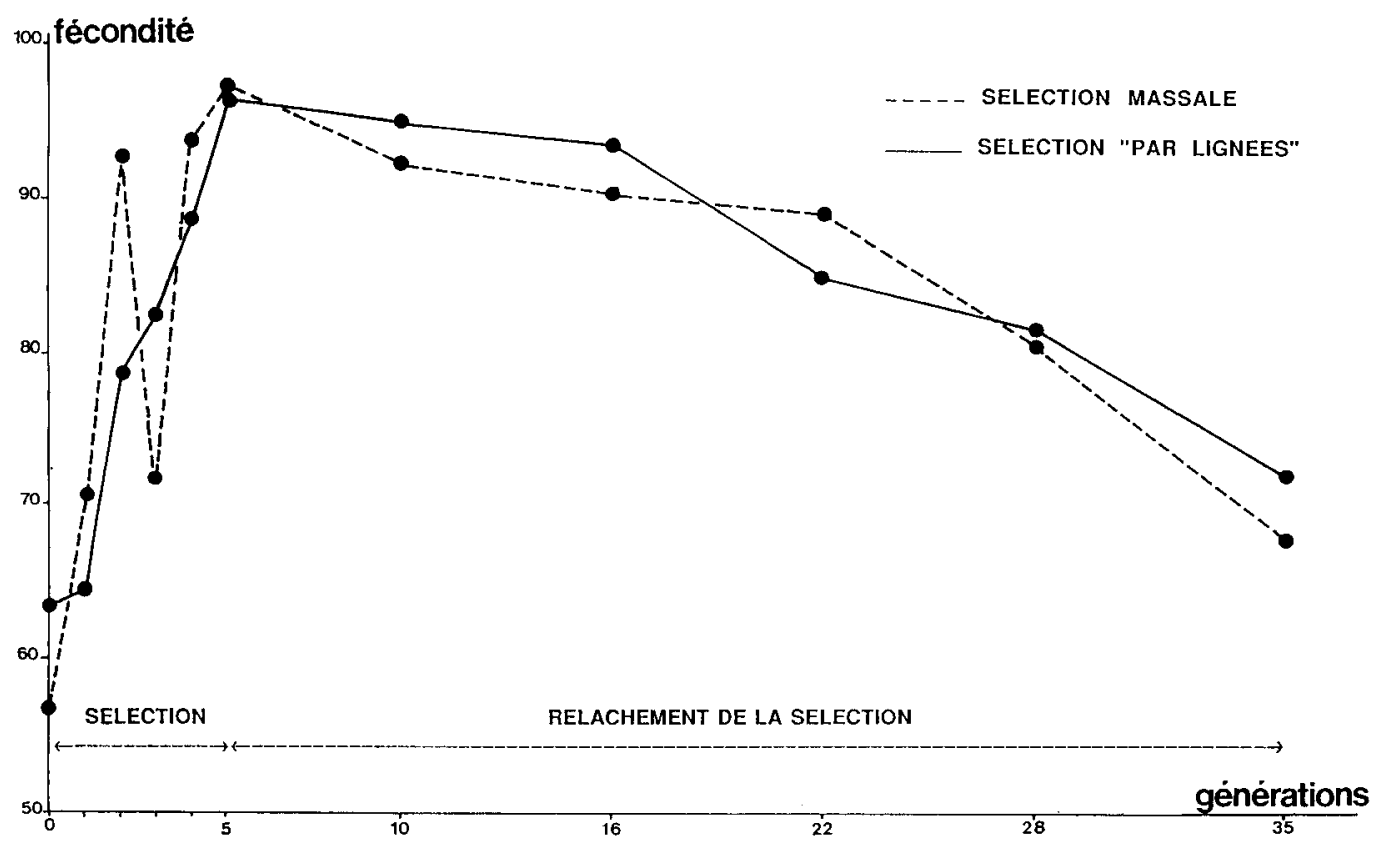

Fig 2. Fécondité de $T$ brassicae après le relâchement de la sélection. 


\section{Capacité de recherche de l'hôte (pression de 50\%)}

Pour ce caractère, la sélection n'a pas été efficace (fig 3 et tableau III). Les réponses obtenues sont bien inférieures aux réponses attendues et les corrélations ne sont pas significatives. Comme précédemment, aucun effet d'hétérosis n'apparaît clairement au cours de la sélection "par lignées".

Cet échec peut être attribué à une pression trop faible, à des conditions de mesure du caractère insuffisamment standardisées ou, plus vraisemblablement, à une surestimation de l'héritabilité.

\section{Fécondité, "capacité de recherche de l'hôte» et index (pression de 66\%)}

Les sélections sur un seul caractère sont destinées à vérifier la répétitivité des résultats précédents et à permettre des comparaisons lors du test de l'efficacité parasitaire de la population sélectionnée pour son index.

Les valeurs $r_{p}$ (coefficient de corrélation entre les 2 caractères) et $\omega$ (importance économique de chaque caractère) entrant dans le calcul de l'index sont les suivantes :

$-r_{p}=0,011$ avec $n=200$, valeur non significative;

$-\omega_{F}=1$

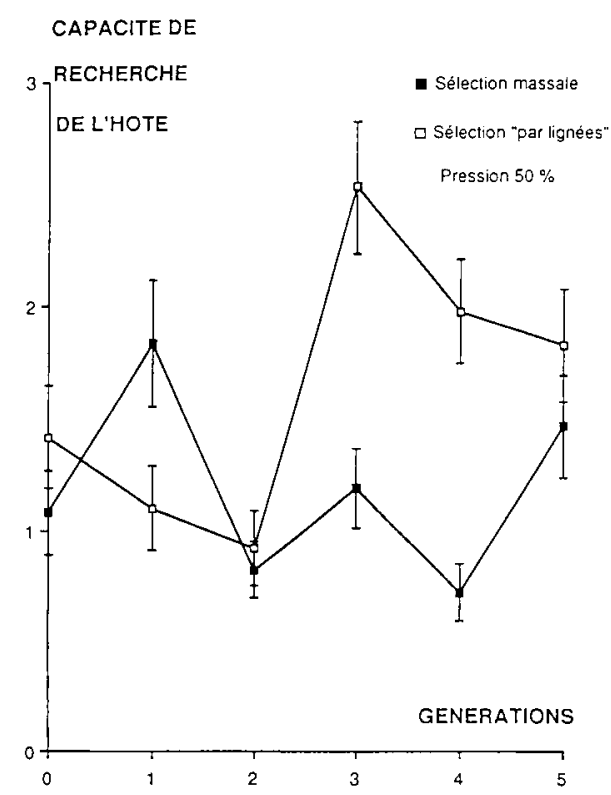

Fig 3. Sélection de la "capacité de recherche de l'hôte» chez $T$ brassicae en 5 générations.

$$
-\omega_{C R}=\frac{\% \text { lignées } C R-\% \text { témoin }}{\% \text { lignées } F-\% \text { témoin }}=0,55
$$

où $\%=$ pourcentage de parasitisme;

lignées = sélection "par lignées" (pression $50 \%) ; C R=$ "capacité de recherche de l'hôte»; $F$ $=$ fécondité.

Nous n'avons pas relevé d'efficacité significative de la sélection, ni pour la fécondité (malgré une amélioration de $32 \%$ ), ni pour la "capacité de recherche de l'hôte", ni pour l'index (fig 4 et tableau III). Les réponses obtenues sont toujours inférieures à celles attendues, les corrélations caractère-génération ne sont pas significatives (sauf pour l'index en retirant le résultat de la $F_{2}$ où a effectivement eu lieu un accident thermique).

En ce qui concerne la «capacité de recherche de l'hôte", la pression de sélection n'était donc pas en cause précédemment. En ce qui concerne la fécondité, cela pose un problème nouveau. La sélection, réussie une année, n'a en effet pas pu être renouvelée 2 ans plus tard à partir des mêmes souches. Deux hypothèses peuvent alors être formulées :
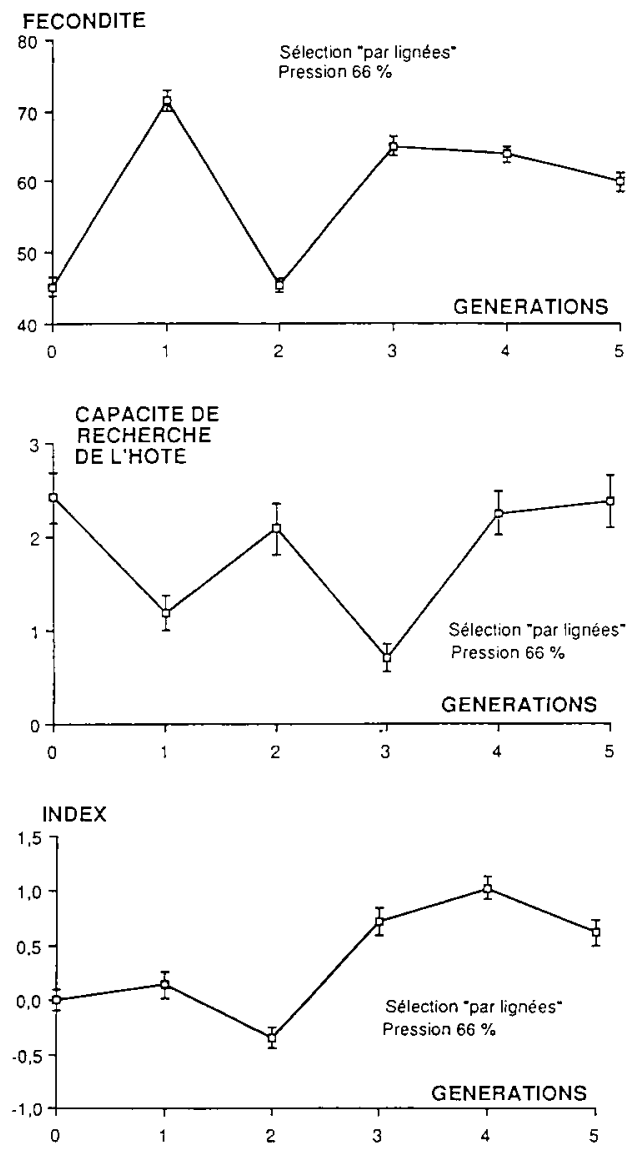

Fig 4. Sélection de la fécondité, de la «capacité de recherche de l'hôte" et des 2 caractères à la fois (index) chez $T$ brassicae en 5 générations. 
- les premières sélections, malgré toutes les apparences, n'ont été qu'un artéfact d'échantillonnage;

- les souches ont subi une sélection homogénéisante durant les 2 années d'élevage.

En tout état de cause, cela montre les difficultés que pourra rencontrer le sélectionneur si cette technique entre dans la pratique de la lutte biologique. Ceci d'autant qu'elle devra être utilisée chaque année pour s'opposer d'une part à l'absence probable de stabilité du caractère sélectionné, d'autre part à la sélection artificielle non intentionnelle (ce qui implique de repartir au moins partiellement de souches nouvelles capturées dans la nature).

\section{Efficacité parasitaire}

Des différences significatives n'ont été relevées que dans 3 cas, à l'aide du test "t" mais pas du test "U". Ceux-ci ne concernent que les populations sélectionnées avec une pression de $50 \%$ (tableau IV) :

- sélection "par lignées" de la fécondité-témoin; en admettant que la sélection ait bien été efficace, on pourrait penser qu'une amélioration du taux de parasitisme a ici été obtenue;

- sélection «par lignées» de la féconditésélection massale de la fécondité; il faut alors admettre que la sélection massale a été efficace mais s'est accompagnée de perturbations (perte de variabilité, par exemple);

- sélection "par lignées" de la "capacité de recherche de l'hôte" -sélection massale de la "capacité de recherche de l'hôte"; ces sélections n'ont pas été efficaces et aucune amélioration du taux de parasitisme n'est d'ailleurs constatée par rapport au témoin, la manipulation de la population "sélectionnée en masse" a par contre pu créer des perturbations susceptibles d'expliquer l'écart constaté.

Nous pouvons remarquer que si les sélections "par lignées" n'ont pas toujours conduit à une amélioration, elles n'ont pas été défavorables. II n'en est semble-t-il pas de même pour les sélections massales, bien que ceci demiande à être confirmé.

\section{DISCUSSION ET CONCLUSION}

Deux types de stratégies sont envisageables pour la lutte biologique à l'aide des Trichogrammes : ou bien on ménage comme actuellement la plus grande variabilité génétique possible, ou bien on sélectionne les individus dont l'efficacité parasitaire est supposée, à tort ou à raison, supérieure. Nous nous sommes ici intéressés aux problèmes posés par cette deuxième possibilité. De telles sélections peuvent être menées soit au laboratoire, comme celles dont nous venons de présenter les résultats, soit sur

Tableau IV. Pourcentages de parasitisme des œufs d'Ostrinia nubilalis (déposés sur du maïs planté sous serres) par $T$ brassicae. Comparaison de l'efficacité de populations sélectionnées, entre elles ou à celles de témoins non sélectionnés. La pression de sélection a été de 50 ou $66 \%$. La comparaison des pourcentages moyens a été effectuée par les test «t" après transformation angulaire et par le test "U» non paramétrique $(n=6$ ou $n=8)$. Trois comparaisons seulement ont fourni des différences significatives : " au seuil de $5 \%$ avec les 2 autres pourcentages, par le seul test "t»; "* au seuil de $5 \%$ avec le pourcentage 53,1 , par le seul test «t».

\section{Témoin Sélection massale Sélection par lignées}

Fécondité (50\%)

Chaque serre est divisée

en 6 parcelles

Capacité de recherche de l'hôte (50\%)

Chaque serre est divisée

en 8 parcelles

Sélection par lignées $(66 \%)$

Chaque serre est divisée

en 8 parcelles

$\begin{array}{lll}16,0 & 16,0 & 51,7^{\star} \\ (0-37,5) & (0-35,7) & (16,7-100) \\ \sigma=21,1 & \sigma=12,1 & \sigma=25,1 \\ 75,0 & & \\ (0-100) & 53,1 & 94,77^{\star \star} \\ \sigma=42,5 & (0-100) & (80-100) \\ & \sigma=27,3 & \sigma=11,9\end{array}$

Fécondité Capacité de recherche de l'hôte Index

$\begin{array}{lll}41,9 & 46,7 & 30,9 \\ (0-100) & (0-100) & (0-66,7) \\ \sigma=29,1 & \sigma=26,1 & \sigma=19,4\end{array}$


le terrain en conditions naturelles ou seminaturelles.

Au laboratoire, les problèmes soulevés par la sélection peuvent être classés en 6 points.

\section{Quel caractère, relativement simple} et impliqué dans la découverte et le parasitisme de l'hôte, faut-il choisir?

Pour dépasser le choix empirique que nous avons fait, il faudrait connaître assez précisément le comportement des Trichogrammes dans le milieu naturel et les facteurs qui limitent le taux de parasitisme. Ceci permettrait de discerner les facteurs qui présentent une certaine constance (une faible sensibilité à une kairomone, par exemple) de ceux trop soumis aux conditions d'une année ou d'une zone géographique relativement réduite (une faible résistance à des températures régnant exceptionnellement, par exemple). Seuls les premiers peuvent être retenus car il paraît utopique de souhaiter une population adaptée à toutes les conditions possibles au niveau d'une vaste zone géographique.

\section{Le choix d'un seul caractère est-il suffisant ?}

Cela simplifierait bien sûr les problèmes mais semble peu probable pour de tels insectes relâchés dans la nature et soumis à de nombreuses contraintes. Si les caractères à améliorer ne sont pas corrélés, il est alors nécessaire de travailler à partir d'un index plus ou moins complexe.

\section{Comment quantifier les caractères ?}

Si ceci peut paraître simple pour la fécondité ou la longévité (il faut malgré tout choisir parmi un certain nombre de conditions possibles, notamment climatiques), c'est plus compliqué pour d'autres caractères. La mesure de la "capacité de recherche de l'hôte", par exemple, peut être effectuée de manières très diverses (petite ou grande cage, observation durant une période plus ou moins longue, hôtes correspondant ou non au ravageur visé, etc) et le risque d'étudier un caractère entièrement artificiel est grand. II serait souhaitable de connaitre la corrélation entre le caractère mesuré et une caractéristique pouvant être appréciée dans la nature.

\section{L'héritabilité des caractères choisis est-elle importante?}

Un caractère ne peut être sélectionné que si sa variabilité génétique est suffisante. Celle-ci doit donc être estimée le plus justement possible. Plusieurs mesures, par différentes méthodes (régression parents-enfants, comparaison des variabilités intra- et interfamiliales), sont alors souhaitables pour éviter les erreurs d'échantillonnage. Encore que ceci n'exclut pas les risques d'erreurs, que certains de nos résultats illustrent peut-être, dus aux effets maternels et à l'utilisation de méthodologies conçues pour les diplodiploïdes et demandant peut-être une légère adaptation aux haplo-diploïdes.

\section{Comment mener la sélection ?}

La méthode choisie doit tenir compte des risques encourus, qui concernent les effets d'entraînement (gènes défavorables liés aux gènes sélectionnés), la perte excessive de variabilité et le déséquilibre du génome. Bien peu de données sont cependant disponibles chez les Trichogrammes.

\section{La population sélectionnée est-elle réellement plus efficace que les autres lorsqu'elle est lâchée contre un ravageur des cultures?}

Même en sélectionnant très efficacement quelques caractères au laboratoire, il n'est pas sûr que la population montre une capacité parasitaire plus élevée dans la nature. Ceci est illustré par certains de nos résultats. On a en effet pu choisir de mauvais caractères, déséquilibrer le génome, entraîner des caractères défavorables, trop diminuer la variabilité.

La sélection sur le terrain où se déroule la lutte biologique, qui n'a jamais encore été tentée, concernerait le caractère complexe "efficacité parasitaire». Elle porterait en fait à la fois sur une meilleure adaptation à des conditions climatiques, une végétation, un hôte (qui ne serait plus celui du laboratoire), et sur une meilleure capacité de découverte des hôtes. Cela permettrait d'éliminer le délicat choix de la nature, du nombre et du mode de mesure des caractères, les études d'héritabilité et l'option pour une technique de sélection. En revanche, on ne connaîtrait ni exactement ce qui a été modifié, ni l'ampleur de la modification. Ces manipulations dans les conditions naturelles diminueraient également les risques de perturbation de la population. Au pire, aucune sélection ne serait obtenue. Nous pourrions procéder de la façon simple suivante : lâcher de Trichogrammes dans plusieurs champs où existent des hôtes potentiels, à partir de quelques points suffisamment distants; 
contrôle à partir du $4^{\mathrm{e}} \mathrm{j}$ après les lâchers des hôtes se trouvant à une certaine distance des points de lâcher; récolte des premiers hôtes parasités; fondation de lignées à partir de ces hôtes infestés qui serviront aux lâchers de l'année suivante. Cette technique retiendrait donc des individus dont les parents ont été capables, dans les conditions naturelles, de découvrir des hôtes à une certaine distance de leur point de lâcher et de les parasiter. Seule l'expérience dira si elle conduit à une réelle amélioration génétique et permettra d'affiner la méthodologie (distance séparant les points de lâcher et les hôtes parasités récoltés, nombre de Trichogrammes lâchés, etc). Un risque devra tout de même être évalué, celui de sélectionner une population adaptée aux conditions climatiques d'une année qui ne se répètent pas l'année suivante.

Aucune sélection menée au laboratoire n'a jusque-là conduit à des insectes (Trichogrammes ou autres espèces) incontestablement plus efficaces en lutte biologique. II ne faut toutefois pas encore conclure à l'impossibilité d'avoir recours à de telles méthodes car bien des recherches restent à effectuer. Le choix entre sélection au laboratoire et sur le terrain paraît cependant être un problème prioritaire à résoudre.

La première méthode présente des avantages (maitrise plus ou moins grande des caractères modifiés, suivi de la sélection) mais aussi d'importants inconvénients (risques d'erreurs portant sur le choix des caractères, de mauvaise appréciation de l'héritabilité, etc). La deuxième méthode ne présente pas les avantages mais, et c'est essentiel, pas non plus les inconvénients de la première. Son déroulement dans les conditions naturelles est un aspect positif supplémen- taire qui peut toutefois être contrebalancé par une adaptation trop stricte à un environnement particulier susceptible de subir des modifications.

Les 2 méthodes créent par ailleurs un excès de travail au moment où l'élevage intensif ou les lâchers de lutte biologique sont déjà très prenants. II faudra donc apprécier si ce surcroît de travail peut apporter suffisamment de bénéfices en termes d'efficacité de la lutte biologique. Les quelques modifications entraînées par l'introduction de la sélection dans le processus d'élevage ont été illustrées dans le tableau V. II faut distinguer entre populations bisexuées pour lesquelles le mélange des lignées conservées est obligatoire et populations thélytoques pour lesquelles ce mélange n'est pas nécessaire (sélection portant sur des clones).

Si elles se révèlent utiles, ces manipulations supplémentaires ne sont bien sûr pas impossibles à réaliser, mais nous pouvons nous interroger sur l'opportunité de les introduire rapidement dans les techniques de lutte biologique. Que représente en effet l'amélioration que l'on peut espérer de la sélection face à celle pouvant provenir de meilleures techniques d'élevage et de lâcher, ou d'une meilleure compréhension de la dynamique des populations?

L'utilisation de populations dont on cherche à conserver la variabilité naturelle n'a certainement pas livré tous ses secrets et ne doit pas être abandonnée. Des priorités sont à respecter. II est d'abord nécessaire de recourir à de telles populations afin d'établir une lutte qui soit sûre. Et ce n'est qu'ensuite que les applications de la sélection, non encore au point mais de toute façon prématurées, pourront peut-être apporter

Tableau V. Processus d'élevage, scindé en plusieurs lignées, des Trichogrammes utilisés en lutte biologique (pas de sélection) et opérations supplémentaires requises en cas de sélection. $c$ : captures; I = lignées; Mél : mélange; Mult : multiplication intensive; Sél : sélection; les flèches dirigées vers le bas indiquent l'introduction de nouvelles lignées dans l'élevage (après captures) ou l'élimination de lignées.

\begin{tabular}{|c|c|c|c|}
\hline $\begin{array}{l}\text { Population } \\
\text { bisexuée }\end{array}$ & 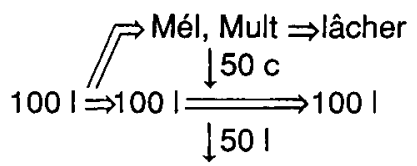 & 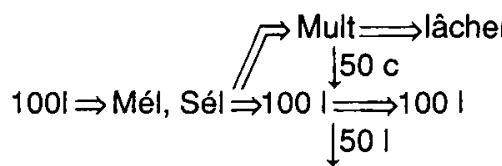 & $100 \mathrm{l} \Rightarrow$ Mél Mult $\Rightarrow$ lâcher, Sél $\Rightarrow 100 \mathrm{I}$ \\
\hline $\begin{array}{l}\text { Population } \\
\text { thélytoque }\end{array}$ & 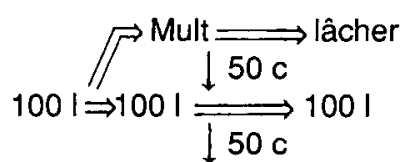 & 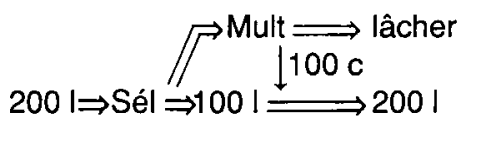 & $100 \Leftrightarrow$ Mult $\Rightarrow$ lâcher, Sél $\Rightarrow 1001$ \\
\hline
\end{tabular}


une nouvelle amélioration. Les connaissances en génétique, actuellement trop faibles, ont donc peut-être le temps de faire des progrès significatifs avant que leur application ne corresponde à un besoin réel.

\section{REMERCIEMENTS}

Nous remercions $M$ Babault, A Babi, J Daumal et $J$ Pizzol pour leur participation aux opérations de sélection et aux essais d'efficacité sous serres.

\section{RÉFÉRENCES}

Ashley TR, Gonzalez D, Leigh TF (1974) Selection and hybridization of Trichogramma. Environ Ento$\mathrm{mol} 3,43-48$

Babi A (1985) Étude de deux espèces de Trichogrammes: Trichogramma maidis Pintureau et Voegelé et $T$ dendrolimi Matsumura (Hym Trichogrammatidae). Choix et essais de sélection de l'espèce la plus efficace pour lutter contre Ostrinia nubilalis Hübner (Lep Pyralidae). Thèse Docteur Ingénieur, Univ Aix-Marseille, $124 \mathrm{p}$

Babi A, Pintureau B (1984) Selection of Trichogramma maidis Pintureau and Voegelé (Hym Trichogrammatidae) fecundity. Abstract Vol, XVII Int Cong Entomol (Hamburg), 795

Bigler F, Baldinger J, Luisoni L (1982) L'impact de la méthode d'élevage et de l'hôte sur la qualité intrinsèque de Trichogramma evanescens Westw. Les Colloques de I'INRA 9, 167-180

Falconer DS (1974) Introduction à la génétique quantitative. Masson, Paris, $284 \mathrm{p}$

Pielou DP, Glasser RF (1951) Selection for DDT tolerance in a beneficial parasite, Macrocentrus ancylivorus Roh. I. Some survival characteristics and the DDT resistance of the original laboratory stock. Can J Zool 29, 90-101

Pintureau B (1987) Systématique évolutive du genre Trichogramma Westwood (Hym Trichogrammati- dae) en Europe. Thèse Doctorat d'Etat, Univ Paris VII, $311 p$

Pintureau B (1988) Genetic variation in the genus Trichogramma Westwood (Hym Trichogrammatidae). Les Colloques de I'INRA, 48, 111-113

Pintureau B, Babault M, Voegelé J (1981) Étude de quelques facteurs de variation de la fécondité chez Trichogramma maidis Pintureau et Voegelé (Hym Trichogrammatidae). Agronomie 1, 315-322

Ram A, Sharma AK (1977) Selective breeding for improving the fecundity and sex-ratio of Trichogramma fasciatum (Perkins) (Trichogrammatidae: Hymenoptera), an egg parasite of lepidopterous hosts. Entomon 2, 133-137

Robertson JG (1957) Changes in resistance to DDT in Macrocentrus ancylivorus Rohw (Hymenoptera : Braconidae). Can J Zool 35, 629-633

Simmonds FJ (1947) Improvement of the sex-ratio of a parasite by selection. Can Entomol 79, 41-44

Szmidt A (1972) Studies on the efficiency of various strains of the parasite Dahlbominus fuscipennis (Zett) (Hymenoptera, Chalcidoidea) under natural conditions. Ekol Pol 20, 299-313

Urquijo P (1944) Ensayo del parasito util Trichogramma minutum en la lucha contra la polilla de la patata. Bol Patol Veg Entomol Agric 13, 281-300

Urquijo P (1946) Selecciòn de estirpes de Trichogramma minutum Riley de maxima efectividad parasitaria. Bol Patol Veg Entomol Agric 14, 199-216

Urquijo Landaluze $P$ (1950) Aplicaciòn de la genetica al aumento de la eficacia del Trichogramma minutum en la lucha biologica. Bol Patol Veg Entomol Agric 18, 1-20

White EB, De Back P, Garber MJ (1970) Artificial selection for genetic adaptation to temperature extremes in Aphytis lingnanensis Compere (Hym Aphelinidae). Hilgardia 40, 161-192

Wilkes A (1942) The influence of selection on the preferendum of a chalcid (Microplectron fuscipennis Zett) and its significance in the biological control of an insect pest. Proc $R$ Soc Lond (Ser B) 130, 400415

Wilkes A (1947) The effects of selective breeding on the laboratory propagation of insect parasites. Proc $R$ Soc Lond (Ser B), 134, 227-245 